formation. Concerning the age of these sandstone rocks a considerable amount of controversy has taken place in recent years, and they have been referred by different authors to the Triassic, the Jurassic, and the Neocomian systems. The fossils found by Overweg and others, however, seem to leave no room for doubt that the real age of the Nubian sandstone is the Cenomanian, or lower portion of the Upper Cretaceous.

Lying upon these sandstones are found great deposits over 600 feet in thickness, consisting of dark green and grey, finely-laminated marls in their lower, and of white, earthy limestones in their upper part. These rocks contain many characteristic Upper Cretaceous fossils, such as Ananchytes ovatus, Ventriculites, and Rudistes. These Upper Cretaceous rocks have been found not only forming the whole southern margin of the Desert, but also rising above the sandy wastes in the hilly masses which form the oases.

The deposits which underlie the greater part of the Sahara appear to be of Tertiary age and referable to the Nummulitic and Miocene periods. There would seem to be no sharp line of demarcation between the Cretaceous and the Tertiary deposits in this area, and in this, as in many other particulars, which are pointed out by Prof. Zittel, the North African formations of these periods remind us of those of the Rocky-Mountain regions of North America.

The older Tertiary deposits of Northern Africa are divided by Dr. Zittel into two members, which he designates the "Lybysche Stufe" and the "Mokattam Stufe." In the lowest of these (the Libysche Stufe) a widelyspread and very characteristic fossil is the Belemnite-like Graphularia descrtorum, Zitt.; many nummulites and other well-marked Eocene fossils also occur.

There appears to be still some doubt as to whether the "Mokattam Stufe" of Dr. Zittel should be classed as Eocene or Oligocene.

In the northern part of the area various freshwater and marine deposits are found which are now referred to the Miocene. No less than sixty-eight forms of marine mollusca have been determined by Dr. Theodor Fuchs as occurring in these beds, and he is led to regard them as indicating a horizon not far removed from that of the Leitha-kalke of the Vienna basin.

The several formations described succeed one another from south to north, this being the direction of the dip of the beds; their relations to one another are well illustrated in the map and sections which accompany the work.

In the midst of the Beharieh oasis a mass of igneous rock is found rising through the midst of the Upper Cretaceous limestones. This rock has been studied by Prof. Zirkel of Leipzic, who pronounces it to be an ordinary plagioclase basalt, very similar in character to that of the Giant's Causeway in Ireland.

Over the whole of these formations the great mass of sands of the Desert is spread, and rises in places into hills several hundreds of feet in height.

In reading this address we cannot but feel that Dr. Zirkel has made admirable use of the collections which Dr. Schweinfurth and others have placed in the museum at Munich; and that by their careful study he has been enabled to clear up many of the difficulties which every one must have felt who has endeavoured to understand the geological structure of the great African continent.

\section{PHYSICS WITHOUT APPARATUS ${ }^{1}$} VIII.

$\mathrm{T}$ the preceding articles of this series we have shown 1 how in every department of physics a large number of instructive experiments may be performed without the aid of any more formal apparatus than the usual domestic

\footnotetext{
${ }^{x}$ Continued from p. $53^{8}$
}

appliances of an ordinary household. There remain to be described a few miscellaneous experiments before concluding the subject.

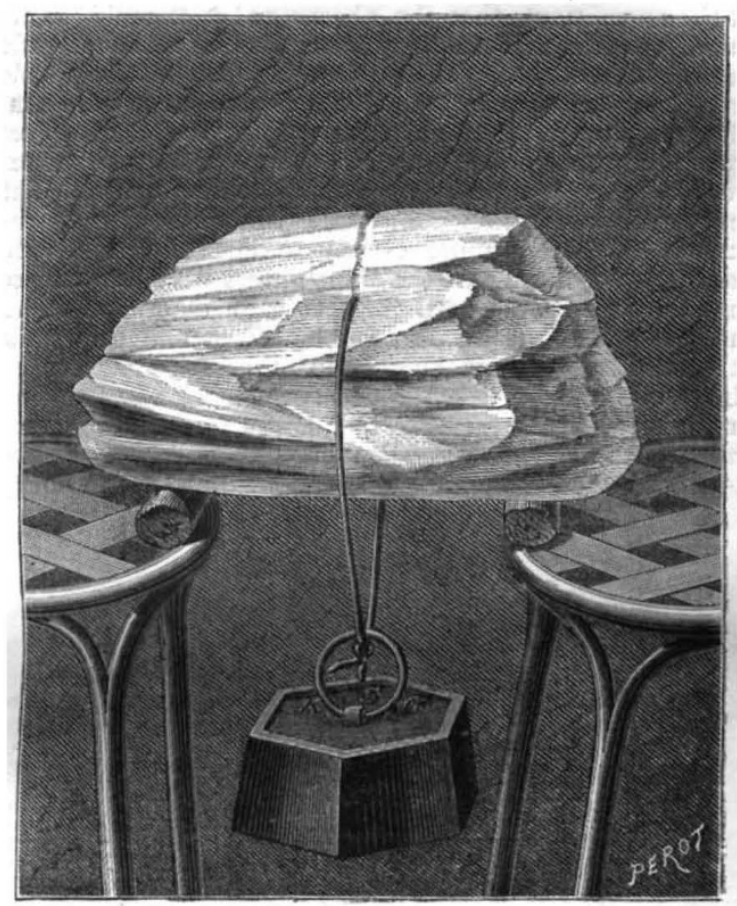

Frg. 25.

Many years ago Prof. Faraday observed that if two pieces of ice are pressed against one another they freeze

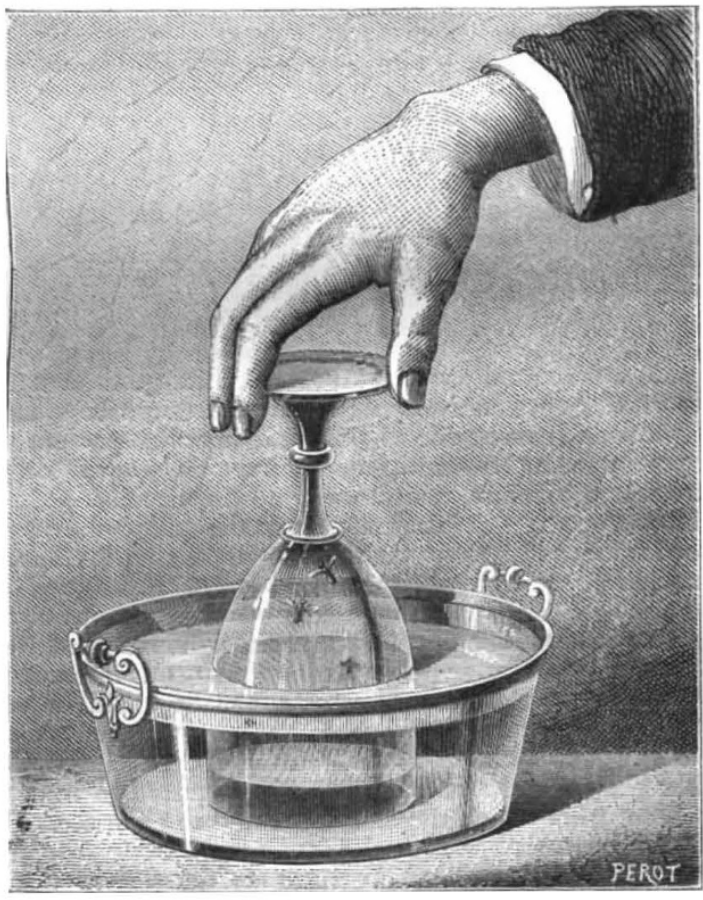

FIG. 26.

firmly together at the point of contact, even though they may themselves be thawing at the surface. To this 
peculiar property of ice he gave the name of regelation. The true explanation of this property was not at once arrived at. From theoretical considerations Prof. James Thomson was led to predict that the application of pressure to ice would lower the temperature of its melting-

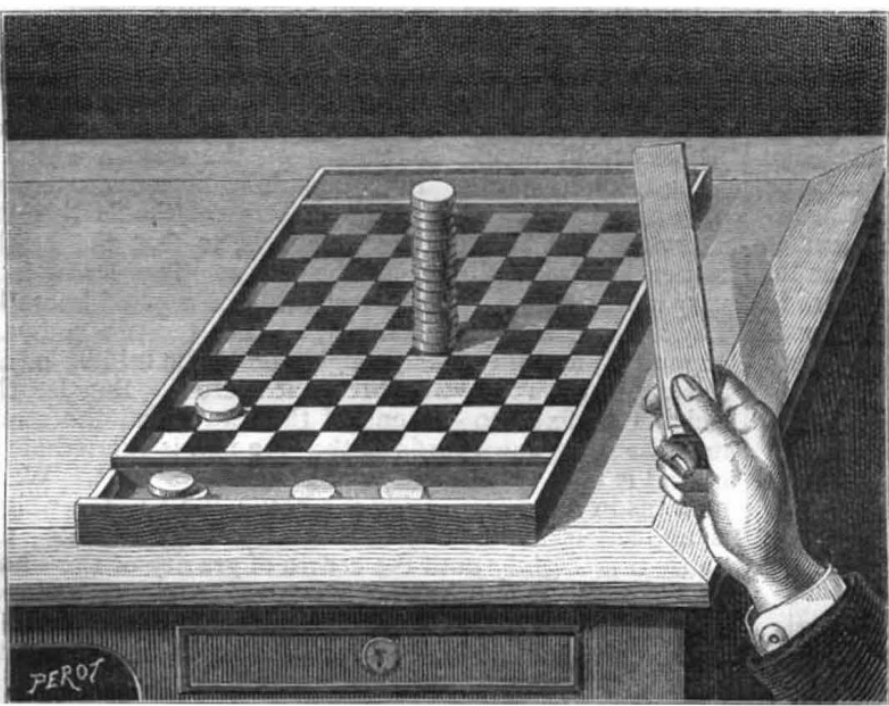

FIG. 27.

point, and cause it to melt even though as cold as, or colder than, the usual "freezing-point." This prediction was afterwards verified by Sir William Thomson, who melted ice by subjecting it to great pressure. More

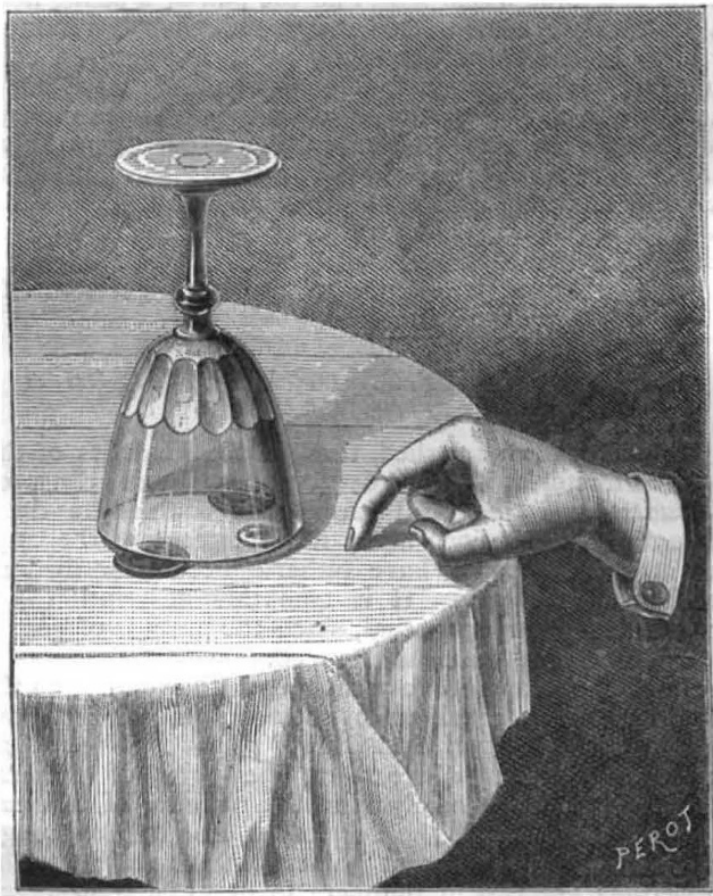

FIG. 28 .

recently Mr. James Bottomley has devised a very beautiful experiment on regelation which requires no special apparatus for its performance. A block of ice (Fig. 25) is placed upon a suitable support, and over it is hung by a copper wire a heavy weight. It is found that in the course of a few hours the weight will have dragged the wire through the ice, as if it were no harder than a piece of cheese, yet that the ice has healed up as fast as the wire cut into it, and that it is still one solid block. This extraordinary fact can be accounted for in the following way. In the neighbourhood of the wire where it passes through the ice the pressures are not uniform, for just below the wire the portions of the ice are under pressure, owing to the pull of the heavy weight, while immediately above the wire the ice is subjected to a stress tending to draw the particles asunder, or, in other words, it is subjected to a pull or "negative pressure." The pressure on the ice under the wire lowers its melting-point, and causes very small quantities of it to melt; these liquid portions immediately are squeezed out, and find their way round the wire to the space above it, where, the pressure being reduced, they again freeze hard.

Our next picture (Fig. 26) is a simple illustration of the principle of the diving-bell. A wine-glass is turned mouth downwards and plunged into a jar of water. The water rises up only a very little way into the mouth of the wine-glass, owing to the air which it contains. The deeper the wine-glass is plunged the more is the air compressed and the higher does the water rise in the miniature bell. To compress the contained air into one-half of its original volume it would be necessary to plunge the wine-glass about thirty-four feet deep into water ; for to halve the volume of the air inside we must double the external pressure. The pressure of the air is already

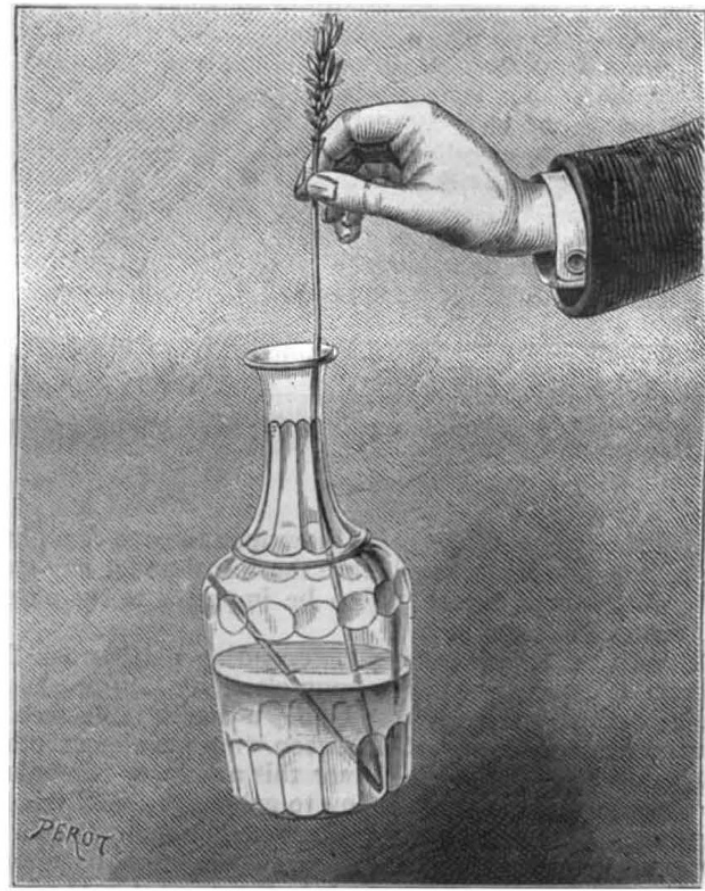

FIG. 29.

about fifteen pounds on every square inch, and to double that pressure requires the additional weight of thirty-four feet of superincumbent water, for that is the depth at which the water itself presses with a force of fifteen 
pounds on each square inch of surface. M. Tissandier, in describing this simple experiment in the pages of $L a$ Nature, has suggested that a few imprisoned flies or other insects may without any cruelty or hurt do duty as divers within the miniature diving-bell, and afford proof that life can go on in the inclosed air even though below the surface of water.

In speaking in a former article of the subject of inertia we mentioned the following familiar trick: a number of the round wooden "men" used in playing the game of draughts are piled up in a column one upon another. If the lowest one of the pile is dextrously hit with the edge of a paper-knife or other suitable article it may be knocked away from under the others without overthrowing the others. Fig. 27 shows how the experiment is arranged, the narrow slip of wood which serves as the lid of the box being here used as the weapon. Beginners in science must not mistake the meaning of the term incrtia as applied to matter. Matter is not in itself lazy or inert. But it possesses the property of mass, and to set mass in motion requires the expenditure of energy. If we skilfully

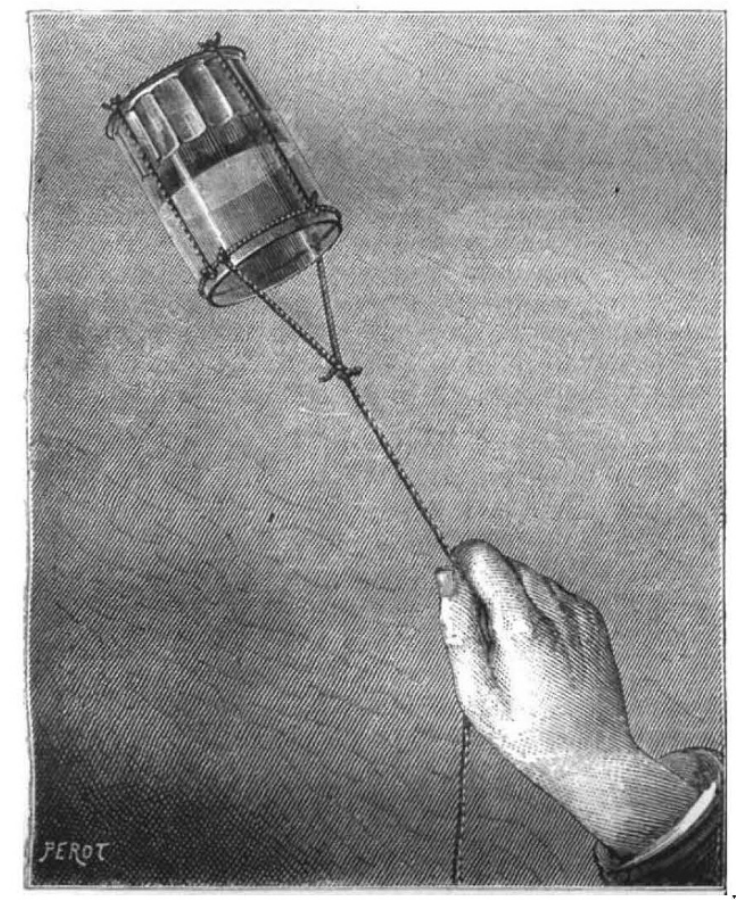

Fig. 30.

spend the energy of the rapid blow upon the one draughtsman, it is knocked away before there is time for any considerable part of the energy to be imparted to the others that are piled upon it.

Another simple experiment, depending partly upon the inertia of matter and partly upon elasticity, is often shown as an after-dinner trick. Upon a linen tablecloth is placed a threepenny-piece between two pennies or other larger and thicker coins. Over this an empty wine-glass is placed, and the puzzle is how to get out the smaller coin without touching the glass. The very simple operation of scratching with the finger-nail upon the cloth, as shown in Fig. 28, suffices to accomplish the trick, for the little coin is seen to advance gently towards the finger until it has moved completely away from under the glass. The fibres of the linen cloth are elastic; when you scratch with your finger-nail they are drawn gently forward until the force of their elasticity becomes too great and they fly back, to be once more drawn forward, again to slip back, and so on. While the fibres are drawn forward slowly, they drag the coin with them to a minute distance. But when the slip occurs and they fly backward, they do so very rapidly, and slip back under the coin before there is time for the energy of their movement to be imparted to the coin to set it in motion. So the coin is gradually carried forward over the surface of the cloth.

We will next give a simple experiment which illustrates the principle that a substance which is very weak in one direction may be very strong in another, the "strength" of the material (that is to say, the resistance it offers before it will break) depending on the way in which a force is applied to it. It is possible to lift a decanter full of water by means of a single straw. To do this the straw must be bent as Fig. 29 shows, so that the weight comes longitudinally upon the straw. The straw is a very weak thing if it has to resist a force applied laterally. Lay a single straw horizontally, so that the two ends are supported, and then hang weights on to the middle of it: a very few ounces will break it across. But let the weights be fixed to one end of the straw, and the straw itself be hung downwards so that the pull is exerted along it, and it will support one or two pounds at least. W'hen bent, as in the figure inside the bottle, most of the weight is applied as a thrust against the end of the straw; the bottle tilts slightly until the centre of gravity of the whole is below the point from which it hangs between finger and thumb; but in this position the sideway thrust against the middle of the straw is very small, and the material is strong enough to stand the strain to which it is subjected lengthways.

Lastly we offer an illustration (Fig. 30) of an experiment known to every schoolboy. A can or jar containing water may be whirled round the head without spilling a single drop, provided the motion be sufficiently rapid. When a moving body is subjected to the constraining action of a force which acts, like the pull of a string held in the hand, towards a fixed centre, the result is a motion around the centre of force. Were it not for the constraining force the moving body would fly away at a tangent ; and to the reaction exerted successively in all directions away from the centre the name "centrifugal force" was formerly given. The water in the can, being heavy, is also subjected to this so-called centrifugal force as it moves around the fixed centre, and hence it does not fall out of the can while passing through the inverted position in the air if its speed be sufficiently accelerated.

\section{THE GERMINATION OF WELWITSCHIA MIRABILIS}

H AVING been supplied, through the kindness of the Director of Kew Gardens, with young seedlings of Welwitschia mirabilis, I have been enabled to draw some fresh conclusions as to the homology of the large leaf structures, which have hitherto been described as persistent cotyledons. It is true this description has been confessedly provisional, since the process of germination has not hitherto been traced.

The seeds germinate in a manner corresponding in the main with that described by Strasburger for Ephedra campylopoda ("Conif. und Gnet.," p. 320). The radicle first breaks through the testa, the point of perforation depending apparently upon the position of the seed during germination. The cotyledons also break through the testa, but at a different point from the radicle. The cotyledons are two in number; in one case I observed three, one being smaller than the others. They free themselves entirely from the seed, and expand to a length of $I$ inch to $I_{2}^{\frac{1}{2}}$ inch, with a breadth of $\frac{1}{8}$ inch, or rather more; it is possible, however, that they may by growth attain a larger size. The cotyledons when expanded are green, though while still in the seed they are yellow. 